\title{
ANTICONSTITUCIONALIDAD DEL SEGURO DE RIESGOS DE TRABAJO Y SU PROBLEMÁTICA ANTE LA REFORMA LABORAL*
}

\author{
UNCONSTITUTIONAL OF THE INSURANCE OF RISKS \\ OF WORK PROBLEMS IN THE LABOR REFORM \\ ANTICONSTITUTIONNALITÉ DE L'ASSURANCE DE RISQUES \\ DE TRAVAIL ET SES PROBLÈMES DANS LA REFORME \\ DU TRAVAIL
}

\section{Verónica Lidia MARTÍNEZ MARTÍNEZ**}

RESUMEN: El presente artículo analiza las distintas consecuencias y prestaciones que originan los riesgos de trabajo, concluyéndose que al cubrirse estas últimas con los recursos acumulados en la cuenta individual, propiedad del asegurado, se contraviene el apartado A, fracciones XIV y XXIX, del artículo 123 constitucional y los tratados internacionales, que señalan a los empresarios como responsables de los riesgos de trabajo. Se culmina con un análisis de la reforma laboral en lo concerniente a las normas procedimentales aplicables a los riesgos de trabajo, ante la importancia que revisten, pues de ellas depende la eficaz y pronta solución de los denominados "conflictos individuales de seguridad social".

Palabras clave: Riesgos de trabajo, incapacidad.

ABSTRACT: This article examines the different consequences and benefits that cause the risk of work, concluding that the latter covered with the funds accumulated in the individual account of the insured property, is contrary to the section A, fractions XIV and XXIX of Article 123 of the Constitution and international treaties, pointing to employers as responsible of risk of work. Culminating with an analysis of the labor

\footnotetext{
* Recibido el 21 de septiembre de 2012 y aceptado para su publicación el 8 de noviembre de 2013.

** Estudiante de doctorado en la Barra Nacional de Abogados (marb_cap@hotmail.com).
} 
Esta revista forma parte del acervo de la Biblioteca Jurídica Virtual del Instituto de Investigaciones Jurídicas de la UNAM

reform, with regard to the procedural rules applicable of risks of work, to the importance, for them depends the effective and early settlement of the so-called "individual social security conflicts."

Key Words: Risks of work, disability.

RÉSUMÉ: L'article analyse les conséquences et des avantages différents qui provoquent des risques professionnels, concluant que ce dernier couverte par les fonds accumulés sur le compte individuel du bien assuré, est contraire à la paragraphe «Une», fractions XIV et XXIX de l'article 123 de la Constitution et les traités internationaux, en montrant que les employeurs responsables de risques professionnels. Culminant avec une analyse de la réforme du travail, en ce qui concerne les règles de procédure applicables à l'risques professionnels, de l'importance, pour eux dépend le règlement efficace et rapide des soi-disant «conflits individuels de sécurité sociale».

Mots-clés: Risques professionnels, incapacité.

SUMARIO: I. Introducción. II. El seguro de riesgos de trabajo en la Ley del Seguro Social. III. Anticonstitucionalidad del seguro de riesgos de trabajo. IV. La reforma a la Ley Federal del Trabajo en materia de riesgos de trabajo. V. Bibliografía.

\section{INTRODUCCIÓN}

$\mathrm{P}$ artiendo de la hipótesis de señalar al seguro de riesgos de trabajo previsto en la Ley del Seguro Social como anticonstitucional, y que ante la problemática que presenta la calificación y valuación de los padecimientos profesionales el acceso al esquema de pensiones que contempla se torna una tarea sumamente complicada e incierta para el asegurado y sus beneficiarios. En el presente artículo se analiza el seguro de riesgos de trabajo a la luz del actual marco jurídico y de las reformas realizadas a la Ley Federal del Trabajo, dada la importancia que revisten, pues los accidentes y enfermedades profesionales no sólo constituyen la más aterradora tragedia que puede limitar o destruir la solidez física, mental y económica del individuo y su núcleo familiar, sino que representan una de las más importantes formas de quebranto económico ante la pérdida de producción, la alteración de los esquemas productivos y los daños en los equipos de fabricación. 
Esta revista forma parte del acervo de la Biblioteca Jurídica Virtual del Instituto de Investigaciones Jurídicas de la UNAM

De acuerdo con los últimos datos estadísticos publicados por la Organización Internacional del Trabajo (en adelante OIT), cada día mueren 6,300 personas a causa de accidentes o enfermedades relacionadas con el trabajo (más de 2.34 millones de muertes por año) y la cifra anual de enfermedades de orden profesional se sitúa en 160 millones, de las cuales 58 millones provocan al menos cuatro días de ausentismo laboral, estimándose además que la carga económica de las malas prácticas de seguridad y salud en el trabajo representan el $4 \%$ del producto interno bruto global de cada año, ${ }^{1}$ lo que repercute de manera significativa en la economía, por lo que se hace necesario el establecimiento de una cultura de la prevención y la adaptación de las asumidas medidas de seguridad y salud en el trabajo o el establecimiento de nuevas disposiciones, a fin de evitar pagar altos precios ante la magnitud y consecuencias derivadas de los riesgos de trabajo.

\section{EL SEGURO DE RIESGOS DE TRABAJO EN LA LEY DEL SEGURO SOCIAL}

Por disposición de los artículos 41, 42 y 43 de la Ley del Seguro Social se define a los riesgos de trabajo como los accidentes y enfermedades a que están expuestos los trabajadores en ejercicio o con motivo del trabajo.

Así, acorde con la Ley del Seguro Social, los riesgos de trabajo se dividen en dos grandes grupos: los accidentes de trabajo y las enfermedades profesionales. Los accidentes de trabajo consisten en toda lesión orgánica o perturbación funcional inmediata o posterior, e incluso la muerte, con motivo de los siniestros originados en el trabajo o en trayecto del domicilio al centro laboral o viceversa (accidente en tránsito), cuya realización es instantánea. En contraste, las enfermedades profesionales se identifican como estados patológicos progresivos, ${ }^{2}$ y respecto de las cuales no basta que un médico las diagnostique para que se consideren de orden profesional, pues debe justificarse, además, su causalidad con las actividades desempeñadas por el asegurado en el centro de trabajo o el medio ambiente a que estuvo expuesto, a través

\footnotetext{
1 Organización Internacional del Trabajo, Seguridad y salud en el trabajo, disponible en bttp:/ / wnw.ilo.org/global/topics/safety-and-health-at-work/lang--es/index.htm [consultada el 29 de octubre de 2012].

2 Tesis I.1o.T.150 L, Semanario Judicial de la Federación y su Gaceta, Novena Época, julio de 2003, t. XVIII, p. 1211.
} 
Esta revista forma parte del acervo de la Biblioteca Jurídica Virtual del Instituto de Investigaciones Jurídicas de la UNAM

de una pericial médica acompañada del desahogo de una pericial técnica en medio ambiente o de una visita armada, ${ }^{3}$ salvo que se trate de las enfermedades de trabajo consignadas en la tabla del artículo 513 de la Ley Federal del Trabajo, a las cuales se les presumen como tales. ${ }^{4}$

En tales circunstancias, atendiendo al tipo de riesgo y a las consecuencias que éstos producen, las prestaciones en dinero se cuantifican de una manera diferente. Así, ante una incapacidad temporal, conceptualizada conforme al artículo 478 de la ley laboral como la pérdida de facultades o aptitudes que imposibilita parcial o totalmente a una persona para desempeñar su trabajo por algún tiempo, y pese a que dicha ley no establece qué debe entenderse por la expresión "algún tiempo", el párrafo primero, fracción I, del artículo 58 de la Ley del Seguro Social lo constriñe al término de cincuenta y dos semanas, durante las cuales el asegurado gozará de las prestaciones en especie consistentes en asistencia médica, quirúrgica y farmacéutica; servicios de hospitalización, aparatos de prótesis y ortopedia, así como la rehabilitación, además del 100\% del salario en que estuviese cotizando al ocurrir el riesgo, como prestación en dinero. Transcurrido el referido plazo, si el operario no ha recuperado la capacidad para trabajar, el Instituto Mexicano del Seguro Social (IMSS) o la Junta Federal de Conciliación y Arbitraje (JFCA) deberán

3 En los últimos años, los tribunales colegiados de circuito en materia laboral conceden el amparo y protección de la justicia federal, para el efecto de que se desahoguen las visitas armadas como uno de los medios idóneos para acreditar la relación causa-efecto entre el medio ambiente en que el asegurado prestó sus servicios y el o los padecimientos que presenta. La visita armada se practica en el centro de trabajo del asegurado, lugar al que acuden el actuario adscrito a la Junta Federal de Conciliación y Arbitraje, las partes, con sus respectivos peritos médicos, y en algunos casos técnicos en medio ambiente para constatar las condiciones ambientales en que se vino desarrollando la actividad o profesión.

4 La tabla contenida en el artículo 513 de la Ley Federal del Trabajo establece el catálogo de enfermedades profesionales de los trabajadores, pero no es limitativa de las enfermedades que dan derecho a obtener indemnización, pues sólo menciona una relación de las provenientes de determinada clase de trabajo. Lo anterior no quiere decir que no existan otras que, sin ser específicas de determinada ocupación, puedan contraer los trabajadores, con ocasión o en ejercicio de su trabajo. Por ello, cuando una determinada enfermedad quede fuera de las enunciadas en la citada tabla, no existe presunción de que dicha enfermedad la adquirió el operario en ejercicio o con motivo del trabajo, del medio ambiente o de las actividades desempeñadas, teniendo este último que acreditar la relación de causa-efecto entre el padecimiento y el trabajo, las actividades desempeñadas o el medio ambiente laboral, pues la fracción XIV del artículo 123 constitucional no limita el concepto de enfermedad profesional, sino que comprende todas aquellas a las que el trabajador esté expuesto en el ejercicio de su trabajo. 
Esta revista forma parte del acervo de la Biblioteca Jurídica Virtual del Instituto de Investigaciones Jurídicas de la UNAM

calificar y valuar, conforme al artículo 514 del código obrero, si se trata de una incapacidad permanente parcial o total.

La incapacidad permanente parcial, definida por el artículo 479 de la ley laboral como la disminución de las facultades o aptitudes de una persona para trabajar, posibilita a que el asegurado, pese a tener daños irreversibles en la salud, pueda ser rehabilitado y reubicado laboralmente en su mismo puesto o en otro acorde con su nueva condición física, sin perjuicio de su salario, ${ }^{5}$ teniendo derecho a las prestaciones en especie establecidas ante una incapacidad temporal, pero atendiendo a la valuación fijada conforme al artículo 514 del código obrero; el monto de las prestaciones en dinero será variable.

Así, ante una valuación definitiva del 25\% de incapacidad, al asegurado se le otorgará una indemnización global equivalente a cinco anualidades; se trata de una valuación definitiva que exceda del $25 \%$, sin rebasar el $50 \%$, le dará derecho a elegir entre la indemnización global o una pensión vitalicia y, finalmente, ante una incapacidad permanente parcial superior al 50\% percibirá una pensión, más un aguinaldo anual equivalente a 15 días del importe de la pensión.

Por su parte, ante una incapacidad permanente total, conceptualizada por el artículo 480 de la Ley Federal del Trabajo como la pérdida absoluta de facultades del trabajador para desempeñar su profesión o cualquier trabajo por el resto de su vida, percibirá las referidas prestaciones en especie y una pensión equivalente al 70\% del salario en que estuviese cotizando, cuando se trate de accidentes de trabajo, y con el promedio del salario base de cotización de las 52 últimas semanas o las que tuviere en caso de una enfermedad profesional, además de un aguinaldo anual equivalente a 15 días del importe de la pensión

Finalmente, en lo concerniente a la muerte, definida por el artículo 343 de la Ley General de Salud como la pérdida de la vida, se otorgarán a los beneficiarios del asegurado o pensionado fallecido a causa de un riesgo de trabajo las referidas prestaciones en especie, además de las prestaciones en dinero

5 Los artículos 498 y 499 del código obrero imponen al patrón la obligación de reponer en su empleo al trabajador que sufrió un riesgo de trabajo, o bien otorgarle el que estuviere en aptitud de desempeñar (reacomodo laboral); derechos que nacen hasta que se determine el grado de incapacidad, ya que esa declaratoria, salvo el caso de incapacidad total permanente, pondrá de relieve si está o no capacitado para desempeñar su labor habitual o si puede desempeñar otra. 
Esta revista forma parte del acervo de la Biblioteca Jurídica Virtual del Instituto de Investigaciones Jurídicas de la UNAM www.juridicas.unam.mx

consistentes en ayuda por gastos de funeral equivalente a 60 días de salario mínimo vigente en el Distrito Federal, la pensión de viudez equivalente al $40 \%$ de la que hubiese correspondido al asegurado fallecido en caso de una incapacidad permanente total, y un finiquito equivalente a 3 anualidades de la pensión otorgada al extinguirse ésta, lo cual acontece cuando el beneficiario contrae nuevas nupcias o entra en concubinato.

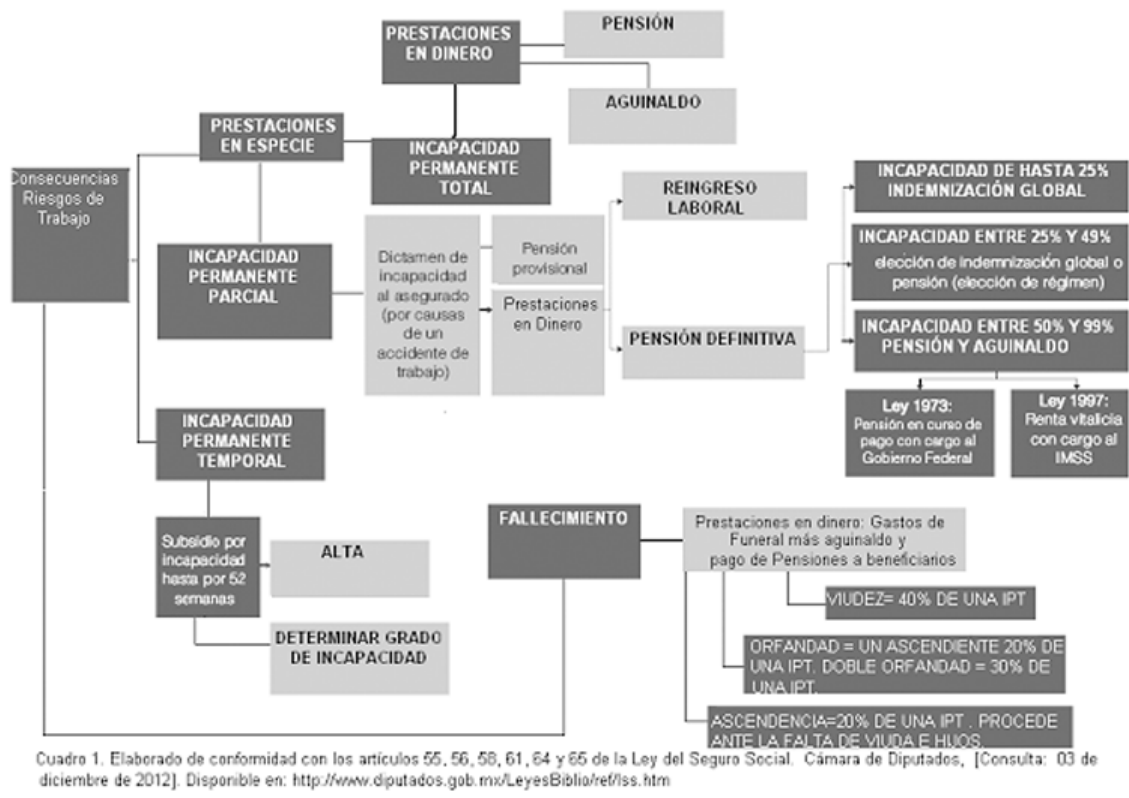

En el caso de los huérfanos, éstos recibirán una pensión equivalente al 20\% de la que hubiese correspondido al asegurado fallecido ante una incapacidad permanente, la cual se incrementará en un 30\% ante una doble orfandad, además de un finiquito equivalente a tres mensualidades de la pensión otorgada al extinguirse ésta, lo cual tiene lugar cuando los huérfanos cumplen 16 años o hasta los 25 años, siempre que se encuentren estudiando en planteles del sistema educativo nacional o desaparezca el estado de incapacidad que hubieran presentado. A falta de viuda, viudo, huérfanos, concubina o concubinario, los ascendientes que hubieran dependido económicamente del asegurado recibirán una pensión equivalente al 20\% de la que hubiese correspondido ante una incapacidad permanente. En todos los casos se otorgará un aguinaldo anual equivalente a 15 días del importe de la pensión correspondiente. Lo 
Esta revista forma parte del acervo de la Biblioteca Jurídica Virtual del Instituto de Investigaciones Jurídicas de la UNAM

anterior se expresa de manera sintética en el cuadro siguiente, elaborado con fundamento en los artículos 55, 56, 58, 61, 64 y 65 de la Ley del Seguro Social.

\section{ANTICONSTITUCIONALIDAD DEL SEGURO DE RIESGOS DE TRABAJO}

Es de vital importancia analizar el tema referente al financiamiento del seguro de riesgos de trabajo, porque de ello dependerá la existencia de recursos suficientes para garantizar el cabal y oportuno otorgamiento y pago de las prestaciones a los asegurados, pensionados y a los beneficiarios de ambos ante la presencia de un riesgo de trabajo, además de cubrir los gastos administrativos que él mismo genera.

Así, en el caso del seguro de riesgos de trabajo existe una excepción al principio de aportación tripartita ${ }^{6}$ característico de los seguros sociales, pues de conformidad con las fracciones XIV, XV y XXIX del artículo 123, apartado A, los patrones son señalados (como producto de una larga e insistente lucha sindical) como únicos responsables de los accidentes y de las enfermedades de los trabajadores, sufridos con motivo o en ejercicio de la profesión o trabajo que ejecuten; estando el empleador, a su vez, obligado a observar los preceptos legales sobre higiene y seguridad en las instalaciones de su establecimiento y adoptar las medidas adecuadas para prevenir accidentes; considerándose como de utilidad pública la expedición de una Ley del Seguro Social que cubra, entre otros seguros, el de enfermedades y accidentes de índole profesional, dándose por terminada aquella época en la que ni por asomo se reputaban como riesgos de trabajo.

En tales circunstancias, si los riesgos de trabajo son responsabilidad de la parte patronal y en cumplimiento a la disposición de la norma fundamental les corresponde asegurar el "bienestar perdido" por el trabajador mediante el pago indemnizatorio, en su caso, genera que las normas que derivan de la norma fundante, y que forman un mismo sistema u orden jurídico, deban

6 El tripartismo, conforme a las previsiones de la Ley del Seguro Social dentro del régimen obligatorio del seguro social, consiste en que las cuotas y las aportaciones son realizadas de manera conjunta por los asegurados, sus patrones y el Estado para financiar las prestaciones contenidas en los seguros de enfermedades y maternidad, invalidez y vida, cesantía en edad avanzada y vejez, con excepción del seguro de riesgos de trabajo, pues de conformidad con la fracción XIV del artículo 123 constitucional, los empresarios son responsables de los riesgos de trabajo. 
Esta revista forma parte del acervo de la Biblioteca Jurídica Virtual del Instituto de Investigaciones Jurídicas de la UNAM

producirse conforme a ella, al ser la que les da sustento jurídico, pues además de representar la unidad a seguir por el sistema jurídico, lo que se traduce en el principio de fundamentalidad constitucional, establece un proceso normativo de creación que lleva a Kelsen a conceptualizarla como la norma que regula la producción de las normas jurídicas generales. ${ }^{7}$

Así, tal y como lo establecieran Ernst Rudolf Bierling, Adolph Merkl y Hans Kelsen, las normas están ordenadas por grados de jerarquía, es decir, cada norma jurídica encuentra su fundamento de validez en otra norma, de tal forma que quedan escalonadas formando una pirámide, ${ }^{8}$ siendo el criterio de jerarquía normativa útil para determinar la validez de una norma inferior, siempre que sea acorde con la norma superior que le da sustento jurídico. ${ }^{9}$ Por ello, dentro del proceso evolutivo graduado de creación normativa, la validez de las normas se puede ponderar a partir de su conformidad con la norma jerárquica superior, siempre que ambas pertenezcan al mismo campo de operatividad normativa. ${ }^{10}$

Atento a lo expuesto, en nuestro país, después de la Constitución, el escalón siguiente lo constituyen los diversos tratados internacionales ${ }^{11}$ ratificados

7 Kelsen, Hans, Teoría pura del derecho, 10a. ed., trad. de Roberto J. Vernengo, México, Porrúa, 1998, pp. 232-234.

8 Senior F., Alberto, Un curso de filosofía del derecho, México, s.e., 1999, p. 87.

9 Kelsen, Hans, op. cit., p. 232.

10 Ibidem, p. 235.

11 El 11 de mayo de 1999, la Suprema Corte de Justicia de la Nación estableció que los tratados internacionales están por encima de las leyes federales mediante la tesis P. LXXVII/99, publicada en el Semanario Judicial de la Federación y su Gaceta, Novena Época, noviembre de 1999, t. X, p. 46, con el rubro "TRATADOS INTERNACIONALES, SE UBICAN JERÁRQUICAMENTE POR ENCIMA DE LAS LEYES FEDERALES Y EN UN SEGUNDO PLANO RESPECTO DE LA CONSTITUCIÓN FEDERAL". Criterio que se considera fortalecido con la resolución recaída a la contradicción de tesis número 293/2011, comúnmente denominada "proyecto Zaldívar", cuya discusión culminó el 3 de septiembre de 2013, al determinar los ministros del alto tribunal que ante la existencia de antinomias entre disposiciones internacionales y constitucionales, en materia de derechos humanos, deben prevalecer las restricciones y limitaciones que tengan los derechos constitucionales sobre los convencionales. Teniendo en cuenta que la seguridad social y los derechos sociales tienen la calidad de derechos humanos por reconocimiento expreso de la comunidad mundial, a través de diversos instrumentos, entre los que destacan: la Declaración Universal de los Derechos Humanos (artículos 22 y 25); la Declaración Americana de los Derechos y Deberes del Hombre (artículo XVI); el Pacto Internacional de Derechos Económicos, Sociales y Culturales (artículo 9o.) y la Convención Americana sobre Derechos Humanos (artículo 26), de acuerdo con la sentencia recaída a la contradicción de tesis número 293/2011, lamentablemente su ejercicio podrá restringirse o limitarse cuando así lo prevenga la 
Esta revista forma parte del acervo de la Biblioteca Jurídica Virtual del Instituto de Investigaciones Jurídicas de la UNAM www.juridicas.unam.mx

por el Senado. Entre los más importantes en materia de accidentes y enfermedades profesionales se encuentran:

- El Convenio 12 relativo a la indemnización por accidentes de trabajo en la agricultura, adoptado el 12 de noviembre de 1921 y ratificado el 1 o. de noviembre de 1937.

- El Convenio 19 relativo a la igualdad de trato entre los trabajadores extranjeros y nacionales en materia de indemnización por accidentes del trabajo, adoptado el 5 de junio de 1925 y ratificado el 12 de mayo de 1934.

- El Convenio 102 sobre la seguridad social (norma mínima), adoptado el 28 de junio de 1952 y ratificado el 12 de octubre de 1961 únicamente en las partes II, III, V, VI y VIII-X.

- El Convenio 134 sobre la prevención de accidentes (gente de mar), adoptado el 30 de octubre de 1970 y ratificado el 2 de mayo de 1974.

- El Convenio 155 sobre seguridad y salud de los trabajadores, adoptado el 22 de junio de 1981 y ratificado el 1o. de febrero de 1984.

- El Convenio 161 sobre los servicios de salud en el trabajo, adoptado el 25 de junio de 1985 y ratificado el 17 de febrero de 1987.

- El Convenio 164 sobre la protección de la salud y la asistencia médica (gente de mar), adoptado el 8 de octubre de 1987 y ratificado el 5 de octubre de 1990.

- El Convenio 167 sobre seguridad y salud en la construcción, adoptado el 20 de junio de 1988 y ratificado el 5 de octubre de $1990 .^{12}$

Convenios que, en su conjunto, en materia de riesgos de trabajo establecen:

norma constitucional, lo cual se considera que vulnera la convencionalidad oficiosa y hace caso omiso del artículo 2o. de la Convención Americana sobre Derechos Humanos, ratificada por nuestro país, que imponen al Estado que se adhiere a un tratado internacional, la obligación de armonizar su derecho interno con el derecho internacional, pero no al revés, como indebidamente lo considera la Corte en la citada contradicción de tesis, ya que la firma de un tratado internacional le impone al Estado signatario el deber de cumplirlo, lo cual se logra mediante el reconocimiento, adopción y constitucionalización de los derechos convencionales en el derecho nacional, pues su inobservancia genera una responsabilidad por parte del Estado obligado. $\mathrm{Al}$ lector interesado en este tema se le recomienda la lectura de la versión taquigráfica de la sesión pública ordinaria del Pleno de la Suprema Corte, celebrada el martes 3 de septiembre de 2013, visible en la página electrónica de la Suprema Corte de Justicia de la Unión: http:/ / wmm. scjn.gob.mx/PLENO/ver_taquigraficas/03092013PO.pdf [consultada: 14 de octubre de 2013].

12 Organización Internacional del Trabajo, "Ratificaciones de México", disponible en: 
Esta revista forma parte del acervo de la Biblioteca Jurídica Virtual del Instituto de Investigaciones Jurídicas de la UNAM

- La responsabilidad primaria del empleador con respecto al pago de prestaciones y la prestación de servicios de salud en el trabajo, siendo acordes con lo establecido en el artículo 123 constitucional, apartado A, fracción XIV.

- Se definen los derechos de los trabajadores y la responsabilidad de los gobiernos para el establecimiento de infraestructuras adecuadas para la práctica de una política de seguridad y salud en el trabajo mediante la legislación, los convenios colectivos y cualquier otro mecanismo aceptable, previa consulta con las organizaciones representativas de los trabajadores y de los empleadores.

- Se establece como una obligación del empleador y de los gobiernos, la prevención de los accidentes de trabajo y las enfermedades profesionales, el control de los peligros del lugar de trabajo, así como el desarrollo de un medio ambiente de trabajo y un trabajo que favorezca la salud de los trabajadores.

Posteriormente, en un tercer plano jerárquico están las leyes federales, dentro de las cuales, respecto del seguro de riesgo de trabajo, se encuentran la Ley del Seguro Social y la Ley Federal del Trabajo, de aplicación supletoria. El primer ordenamiento dispone en su artículo 70 que las prestaciones del seguro de riesgos de trabajo, inclusive los capitales constitutivos de las rentas líquidas al fin de año y los gastos administrativos, deberán cubrirse íntegramente con las cuotas que para este efecto aporten los patrones, enfatizándose así la obligación constitucional de los empleadores de responsabilizarse de los riesgos de trabajo.

En esta misma tesitura, el artículo 53 de la Ley del Seguro Social preceptúa que aquel patrón que haya asegurado a los trabajadores a su servicio contra riesgos de trabajo quedará relevado del cumplimiento de las obligaciones derivadas de un accidente o enfermedad profesional, asumiendo el IMSS la calidad de patrón, al sufragar la pretensión mediante un sistema que consiste en el pago de pensiones.

Así, por disposición de los artículos 58 y 64 de la Ley del Seguro Social, sólo podrán acceder a una pensión derivada de un riesgo de trabajo aquellos asegurados que siendo víctimas del siniestro contraten los seguros de renta

http:// www.ilo.org/dyn/normlex/es/f?p=1000:11200:0::NO:11200:P11200_COUNTRY_ID:102 764 [consultada: 13 de noviembre de 2012]. 
Esta revista forma parte del acervo de la Biblioteca Jurídica Virtual del Instituto de Investigaciones Jurídicas de la UNAM

vitalicia y sobrevivencia, que cubrirá, en caso de fallecimiento del pensionado, las prestaciones económicas a sus deudos. En la contratación del seguro de renta vitalicia y sobrevivencia, primero el IMSS calculará el monto constitutivo o prima que ha de pagarse a la institución de seguros privada que elijan el trabajador y sus beneficiarios; acto seguido, al monto se le deberá restar el saldo acumulado en la cuenta individual del asegurado, y la diferencia positiva será la suma asegurada, que deberá pagar el IMSS a la compañía de seguros.

En tales condiciones, de acuerdo con el texto constitucional y los artículos 53 y 70 de la Ley del Seguro Social, el IMSS continúa recibiendo las cuotas patronales que deberán emplearse para financiar las prestaciones y gastos administrativos que la operación del seguro de riesgos de trabajo genera; pero por disposición de los artículos 58 y 64 de la Ley del Seguro Social, remite al asegurado con las administradoras de fondos para el retiro y compañías aseguradoras para que sean éstas las que les otorguen las prestaciones del seguro de riesgo de trabajo con los recursos depositados en la cuenta individual, los que por disposición del artículo 159 de la Ley del Seguro Social son propiedad de los asegurados, resultando por consecuencia que al destinarse los recursos de la cuenta individual para el financiamiento y otorgamiento de las prestaciones del seguro de riesgos de trabajo, los artículos 58 y 64 de la Ley del Seguro Social contradicen el apartado A, fracción XIV, del artículo 123 constitucional y los referidos tratados internacionales, que señalan a los empresarios como responsables de los riesgos de trabajo que se presenten.

Sobre este tenor, debemos señalar que el artículo 23 del Reglamento de la Ley de los Sistemas de Ahorro para el Retiro dispone la apertura de una cuenta individual para cada asegurado en las administradoras de fondos para el retiro, la cual se integra por las subcuentas de retiro, cesantía en edad avanzada y vejez, vivienda y aportaciones voluntarias (en algunos casos), con sus respectivos rendimientos, en las cuales se depositan las cuotas obrero-patronales y gubernamental que, ante el cumplimiento de una serie de requisitos por parte de los asegurados, servirán para que a los que hayan cotizado en términos de la actual Ley del Seguro Social se les devuelva el seguro de retiro, ${ }^{13}$ las aportaciones

13 En lo concerniente al retiro, tenemos que su instauración en la Ley del Seguro Social obedece a la necesidad de fomentar el ahorro de los trabajadores, ya que de acuerdo con las estadísticas, México es un país que no ahorra por múltiples factores, tanto debido a su natural idiosincrasia como al hecho de que nunca se han buscado ni propiciado mecanismos de ahorro efectivos y eficaces, motivos todos ellos que llevaron a la implementación de la aportación 
Esta revista forma parte del acervo de la Biblioteca Jurídica Virtual del Instituto de Investigaciones Jurídicas de la UNAM

existentes en la subcuenta de vivienda ${ }^{14} \mathrm{y}$ disfruten de una pensión de cesantía o de vejez con los recursos existentes en la subcuenta de cesantía y vejez, los que al ser "tomados" por el IMSS para cubrir las prestaciones del seguro de riesgo de trabajo, generan que al asegurado se le prive de su patrimonio, transgrediéndose el segundo párrafo del artículo 14 constitucional, además de ponerse en riesgo el financiamiento del seguro de cesantía y de vejez.

Ante el desvío de los recursos del seguro de cesantía y de vejez para enmendar los daños originados por un riesgo de trabajo, ello con el patrimonio del asegurado (afectado), implica regresar a aquella época en que los accidentes y enfermedades de trabajo eran responsabilidad del trabajador, perdiéndose de vista que ante la imposibilidad de un asegurado para trabajar se le otorgan tratamientos jurídicos diversos, los cuales atienden a la causa que los provoca.

Así, el seguro de riesgos de trabajo ampara las contingencias relativas a los accidentes y enfermedades sufridos por los asegurados en ejercicio o con motivo de su trabajo, mediante el otorgamiento de prestaciones graduadas en función del tipo de consecuencia producida en la integridad del asegurado. Por el contrario, el seguro de cesantía en edad avanzada y vejez se encuentra sujeto a la reunión de diversos requisitos, tales como el cumplimiento de periodos de espera y semanas de cotización reconocidas por el IMSS, una edad establecida, estar dentro del periodo de conservación de derechos, la ausencia de un trabajo remunerado en el caso de la cesantía, además de implantarse un modelo de capitalización individual, donde la solidaridad desaparece, ya que lo prioritario atiende a la cantidad de dinero que en su vida laboral acumu-

obligatoria de los patrones con el $2 \%$ sobre los salarios por concepto de retiro, resultando por ello que, al adquirir los asegurados el derecho a disfrutar de una pensión de cesantía en edad avanzada o de vejez, tengan expedito su derecho a que se les entreguen los fondos de la subcuenta de retiro en una sola exhibición, pues no tienen por finalidad financiar ningún tipo de pensión o beneficio, además de no guardar relación con los seguros de cesantía en edad avanzada y vejez.

14 El Infonavit tiene la obligación de entregar a los asegurados todos los recursos que hayan acumulado en la subcuenta de vivienda, lo anterior en cumplimiento al Decreto que reforma la Ley del Infonavit y la jurisprudencia emitida por los tribunales colegiados, bajo el rubro "SUBCUENTA DE VIVIENDA. LA OBLIGACIÓN DE DEVOLVER LOS FONDOS ACUMULADOS EN ÉSTA, SURGE DE MANERA CONCOMITANTE Y CONSECUTIVA A LA TRANSFERENCIA QUE EL INFONAVIT DEBA HACER DE ÉSTOS A LA AFORE CORRESPONDIENTE, AUN CUANDO NO SE ESPECIFIQUE QUE EL PAGO RELATIVO SE HAGA PREVIA TRANSFERENCIA DE ELLOS", tesis: I.13o.T. J/14, Semanario Judicial de la Federación y su Gaceta, Novena Época, noviembre de 2010, p. 1409. 
Esta revista forma parte del acervo de la Biblioteca Jurídica Virtual del Instituto de Investigaciones Jurídicas de la UNAM

le el asegurado en la cuenta individual, la cual servirá para que cuando éste concluya su vida activa laboral, y una vez llenados los requisitos legales, se le otorgue una pensión de cesantía o de vejez que le permita pasar los últimos años de existencia con los mínimos satisfactores, afrontando la contingencia social del retiro. ${ }^{15}$

De esta forma, al financiarse con los recursos acumulados en la cuenta individual las prestaciones del seguro de riesgos, que en aras de la subrogación prevista en el artículo 53 de la Ley del Seguro Social debieran cubrirse por el IMSS con las cuotas patronales del seguro de riesgos, sin la intervención de ninguna institución privada, igualmente se contraviene la fracción XXIX del artículo 123 constitucional en su apartado A, que distingue a los riesgos de trabajo de la cesación involuntaria del trabajo y de la vejez, pues sus orígenes, principios, fundamentos, prestaciones, requisitos de obtención, regímenes financieros y acreditamiento son totalmente distintos.

De acuerdo con datos estadísticos, de los 112,336,538 habitantes que representa la población total del país, ${ }^{16}$ el número de asegurados que al 31 de julio de 2013 se encontraba vigente en el IMSS y protegido ante las contingencias del seguro de riesgos de trabajo fue de $16,362,732,{ }^{17}$ lo que representa aproximadamente una cobertura del $14.56 \%$, lo que viene a evidenciar que el número de personas protegidas contra este tipo de infortunios es muy bajo. El $44.5 \%$ de los asegurados totales se encuentra facultado para elegir entre los beneficios de pensión de la Ley del Seguro Social de 1973 y de la Ley del Seguro Social de 1997, en tanto que los asegurados sin derecho a elección de régimen representan el restante $55.5 \%$, quienes tendrán que sufragar el pago de las prestaciones por riesgos de trabajo con sus cuentas individuales. ${ }^{18}$

En el siguiente cuadro se puede apreciar que el factor que tiene mayor repercusión en la magnitud de las sumas aseguradas que cubre el IMSS en

15 Instituto Mexicano del Seguro Social, Ley del Seguro Social, México, IMSS, 2000, p. 27.

16 Instituto Nacional de Estadística y Geografía, Censo de población 2010, México, INEGI, disponible en: http://mmm.inegi.org. $m x$ / default.aspx? [consultada el 11 de octubre de 2013].

17 Secretaría del Trabajo y Previsión Social, Número total de asegurados, México, STPS, 31 de julio de 2013, disponible en: http:/ / mmw.stps.gob.mx/bp/seccion/conoce/areas_atencion/areas_aten cion/web/menu_infsector.html [consultada el 11 de octubre de 2013].

18 Instituto Mexicano del Seguro Social, "Informe al Ejecutivo Federal y al Congreso de la Unión sobre la situación financiera y los riesgos del Instituto Mexicano del Seguro Social 2012-2013", México, IMSS, 2012-2013, disponible en: http:// mmm.imss.gob.mx/estadisticas/Docu mentos/20122013/informecompleto.pdf [consultada el 11 de octubre de 2013]. 
Esta revista forma parte del acervo de la Biblioteca Jurídica Virtual del Instituto de Investigaciones Jurídicas de la UNAM www.juridicas.unam.mx

materia de riesgos de trabajo lo constituyen los saldos acumulados en la cuenta individual propiedad de los asegurados, ante una menor acumulación de fondos, causada ya sea por un aumento en las tasas de comisión que cobran las administradoras de fondos para el retiro o por una disminución de las tasas de rendimiento de cualquiera de las subcuentas.

Cuadro 2. Elementos que inciden en el costo de las sumas aseguradas

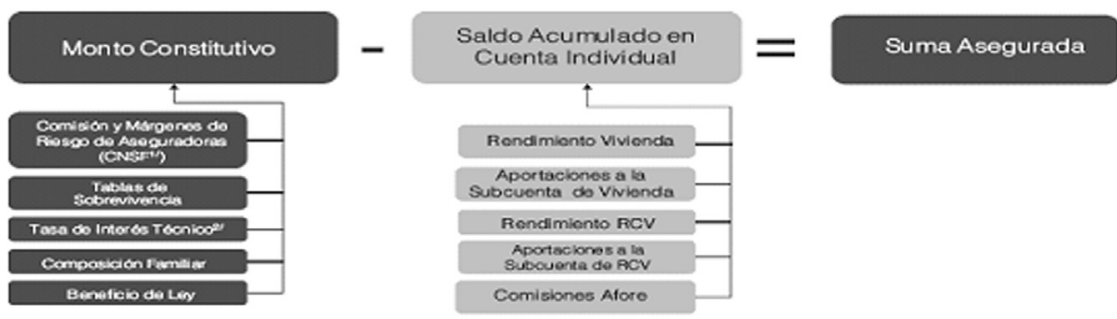

"Comieion Nacional de Seguree y fianzas.

" La tasa do interes ténico corrospondo a la tasa do dosouonto que so utiliza para ol odvculo do la anualidod que sirvo do boso para la easmacion do los montos constitutivos.

Fuonte: DF, ImsS.

Por su parte, en la siguiente gráfica se observa que el área que se encuentra entre la línea de la prima de gasto anual del monto constitutivo y la línea de la prima de gasto anual de las sumas aseguradas constituirá la parte de los montos constitutivos que se estima serán financiados en el seguro de riesgos de trabajo con el importe de las cuentas individuales, en contravención a las fracciones XIV y XXIX del apartado A del artículo 123 constitucional.

\section{Cuadro 3}

Financiamiento de los montos constitutivos por los saldos acumulados en las cuentas individuales, Seguro de Riesgos de Trabajo (gasto en porcentaje de los salarios de cotización)

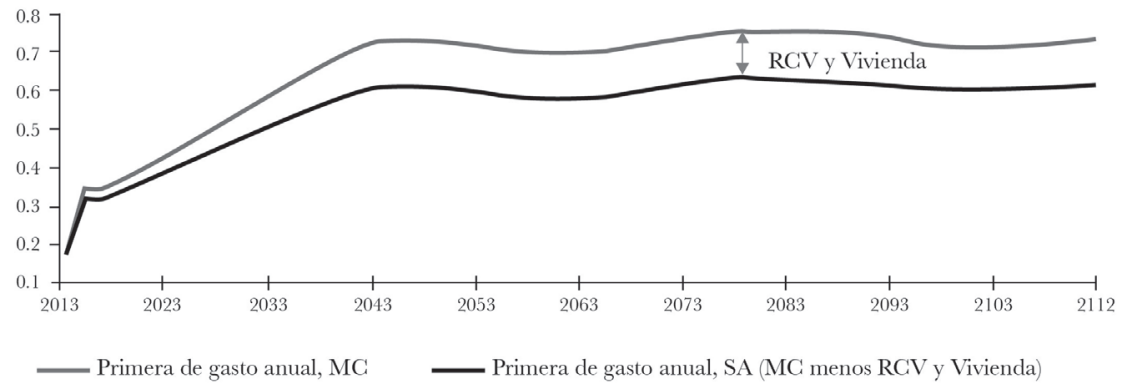

MC-Monto constitutivo, SA-Suma asegurada RCV-Subcuenta de Retiro, Consulta en Edad Avanzada y Vejez; Vivienda-Subcuenta de Vivienda.

Fuente DF, IMSS. 
Esta revista forma parte del acervo de la Biblioteca Jurídica Virtual del Instituto de Investigaciones Jurídicas de la UNAM

Prosiguiendo con el principio de jerarquía normativa, el escalón siguiente es el de las normas reglamentarias relativas al seguro de riesgos de trabajo, dentro de las cuales destacan: el Reglamento Interior del Instituto Mexicano del Seguro Social, el Reglamento de Prestaciones Médicas del Instituto Mexicano del Seguro Social, el Reglamento de la Ley del Seguro Social en Materia de Afiliación, Clasificación de Empresas, Recaudación y Fiscalización, y los diversos reglamentos en materia de seguridad e higiene en el trabajo, los cuales, en su conjunto, establecen que los patrones son responsables no sólo de los riesgos de trabajo sufridos por el operario, sino de la prevención de los mismos.

Finalmente, en el grado más bajo de la pirámide jerárquica se encuentran las normas individualizadas, consistentes en las resoluciones administrativas emitidas por el IMSS, los laudos dictados por la Junta Federal de Conciliación y Arbitraje (JFCA), así como las sentencias emitidas por los tribunales colegiados en materia de trabajo al recurrirse el laudo en vía de amparo directo, las cuales constituyen a favor de los individuos verdaderos derechos subjetivos, pues determinan con base en periciales médicas el tipo de consecuencia que presenta el asegurado y el monto de las prestaciones en dinero que percibirá, las cuales, en el caso de que se determine su procedencia conforme a la actual Ley del Seguro Social, serán cubiertas por el asegurado con los recursos de la cuenta individual.

\section{LA REFORMA A LA LEY FEDERAL DEL TRABAJO EN MATERIA DE RIESGOS DE TRABAJO}

El 13 de noviembre de 2012 el Senado aprobó la reforma a la Ley Federal del Trabajo, hecho que, aunado a la aprobación dada con anterioridad por la Cámara de Diputados, completó el proceso legislativo, enviándose al Ejecutivo para su promulgación y publicación. La reforma laboral, publicada en el Diario Oficial de la Federación del 30 de noviembre de 2012, tiene un impacto fundamental en materia de riesgos de trabajo, no sólo en lo referente a la calificación y valuación de los padecimientos de índole profesional, sino también en la parte procesal.

Así, los artículos 513 y quinto transitorio de la reforma a la Ley Federal del Trabajo disponen que la Secretaría del Trabajo y Previsión Social, previa opi- 
Esta revista forma parte del acervo de la Biblioteca Jurídica Virtual del Instituto de Investigaciones Jurídicas de la UNAM

nión de la Comisión Consultiva Nacional de Seguridad y Salud en el Trabajo, en un término de seis meses contados a partir de la entrada en vigor del referido decreto, expedirá las tablas de enfermedades de trabajo y de valuación de las incapacidades permanentes resultantes de los riesgos de trabajo, las cuales ya no aparecerán en el texto de la Ley Federal del Trabajo. En adelante serán publicadas en el Diario Oficial de la Federación, ${ }^{19}$ lo cual estimamos que obedece a la necesidad de contar con un mecanismo que permita con una mayor periodicidad la revisión, modificación y fácil adecuación de las referidas tablas, las cuales no corresponden con la mundialización, el rápido ritmo de cambio en el lugar de trabajo, las nuevas tecnologías, factores de riesgo y avances científicos, evitándose la tardada y conflictiva reforma del código obrero en términos del artículo 72 constitucional.

Esperemos que la medida adoptada sea benéfica por el bien de todos, aunque no debemos perder de vista el evidente incumplimiento de la Secretaría del Trabajo y Previsión Social (en adelante STPS) a los artículos 515 y Cuarto Transitorio del decreto por el que se reforman, adicionan y derogan diversas disposiciones de la Ley Federal del Trabajo, pues respecto del primer dispositivo legal, ha sido omisa en la realización de investigaciones y estudios necesarios a fin de que el presidente de la República pueda iniciar la adecuación periódica de las tablas de enfermedades de trabajo y de valuación de las incapacidades permanentes resultantes de los riesgos de trabajo conforme al progreso de la medicina del trabajo, lo cual ha generado que, en detrimento de los derechohabientes, hasta el día de hoy continúen vigentes las antiguas, incompletas y anacrónicas tablas consignadas en los artículos 513 y 514 de la Ley Federal del Trabajo, dado que la STPS no realizó su actualización dentro del término de seis meses contados a partir del 1o. de diciembre de 2012, fecha en que entró en vigor el referido decreto, como lo dispone el inobservado artículo cuarto transitorio.

De acuerdo con lo anterior, todo parece indicar que fue un desacierto de la reforma laboral mantener intacto el texto del artículo 515 del código obrero, cuando históricamente se ha demostrado la negligencia, por parte de la STPS, en la realización de investigaciones y estudios necesarios, encaminados a lo-

19 Decreto por el que se reforman, adicionan y derogan diversas disposiciones de la Ley Federal del Trabajo, Diario Oficial de la Federación, México, núm. 23, primera sección, 30 de noviembre de 2012, pp. 61 y 91. 
Esta revista forma parte del acervo de la Biblioteca Jurídica Virtual del Instituto de Investigaciones Jurídicas de la UNAM

grar que el presidente de la República pueda iniciar ante el Poder Legislativo la adecuación periódica de las tablas a que se refieren los artículos 513 y 514 de la Ley Federal del Trabajo. Enfatizamos esta postura porque a más de medio siglo de la aprobación del código de trabajo, ningún titular del Poder Ejecutivo Federal intentó actualizar las multicitadas tablas, no obstante que representaba una obligación que debía cumplir y que, lamentablemente, en el presente se sigue incumpliendo, siendo prueba de ello la inobservancia al policitado artículo cuarto transitorio.

Únicamente se obtendrán buenos frutos cuando la Secretaría del Trabajo y Previsión Social cumpla con la tarea encomendada no sólo de expedir el catálogo de enfermedades de trabajo, sino de actualizarla para que la misma reconozca desde enfermedades causadas por agentes químicos, físicos y biológicos, hasta las de origen respiratorio y de la piel, enfermedades infecciosas o parasitarias, aquellas causadas por el VIH/SIDA, trastornos del sistema osteomuscular, cáncer profesional, trastornos mentales y del comportamiento, ${ }^{20}$ evitándose así que al asegurado se le traslade toda la carga probatoria de acreditar la relación de causa-efecto cuando una enfermedad no se encuentre tipificada ni valuada en las referidas tablas, en cuya expedición y actualización la reforma laboral debió contemplar la participación de las sociedades o de médicos especialistas en medicina del trabajo, al ser necesario tener conocimientos sobre los aspectos médicos y las condiciones del trabajo, los aspectos técnicos de la patología y la medicina del trabajo, los avances en la medicina, la psicología, la ergonomía y las cuestiones relativas a la protección de la salud de los trabajadores.

En lo concerniente a la parte procesal, derivado de la reforma laboral al capítulo XVIII denominado "De los procedimientos especiales", se adiciona

20 La "Lista de enfermedades profesionales" de la Organización Internacional del Trabajo, revisada en 2010, refleja los últimos avances en la identificación y el reconocimiento de las enfermedades profesionales, al incluir una serie de enfermedades profesionales reconocidas internacionalmente e incluir puntos abiertos que permiten el reconocimiento del origen profesional de enfermedades que no figuran en la lista de enfermedades, siempre y cuando se haya establecido un vínculo entre la exposición a los factores de riesgo que resulte de la actividad laboral y las enfermedades contraídas por el trabajador. La referida lista puede consultarse en: Organización Internacional del Trabajo, Lista de enfermedades profesionales (revisada en 2010). Identificación y reconocimiento de las enfermedades profesionales: criterios para incluir enfermedades en la lista de enfermedades profesionales de la OIT, Ginebra, Oficina Internacional del Trabajo, 2010, Serie Seguridad y Salud en el Trabajo, núm. 74. 
Esta revista forma parte del acervo de la Biblioteca Jurídica Virtual del Instituto de Investigaciones Jurídicas de la UNAM

una primera sección en la que se regulan los llamados "conflictos individuales de seguridad social", terminología que consideramos equívoca por involucrar el concepto filosófico de seguridad social, la cual, al ser un medio de asistencia, que brinda a la población de manera general prestaciones que sólo el Estado y, en su caso, el organismo asegurador soportan, su extensión y prolijidad con otras ramas de la ciencia es demasiado amplia, pues no se limita a problemas económicos y laborales, sino que cubre otras necesidades, tales como las prestaciones sociales con amplísimas y diversas funciones, ${ }^{21}$ involucrándose aspectos que van más allá de la simple referencia a prestaciones derivadas del régimen del seguro social, aquellas que corresponde cubrir al Instituto del Fondo Nacional de la Vivienda para los Trabajadores, a las administradoras de fondos para el retiro y las que resulten aplicables en virtud de contratos colectivos de trabajo o contratos-ley que contengan beneficios en materia de seguridad social, establecidas en el artículo 899-A de la reforma realizada a la Ley Federal del Trabajo.22

Asimismo, debemos señalar que a pesar de que los artículos 899-A a 899-G de la reforma laboral establecen las normas procesales que regulan la competencia por razón de territorio de las juntas especiales de la Federal de Conciliación y Arbitraje para conocer de este tipo de conflictos, además de precisarse quiénes se encuentran legitimados para plantearlos y establecer los requisitos que deben cumplir las demandas, así como en qué casos la carga de la prueba corresponde a los organismos de seguridad social, carece de normas particulares respecto al desarrollo del procedimiento en los referidos conflictos individuales de seguridad social y de los requisitos especiales que debe contener el laudo que llegue a dictarse, ${ }^{23}$ por lo que estimamos que ante dichas lagunas su regulación se debe acoger a las disposiciones generales para la tramitación de los procedimientos especiales.

En vista de lo anterior, y de acuerdo con el artículo 899-A de la reforma realizada a la Ley Federal del Trabajo, la reclamación de las prestaciones derivadas del seguro de riesgos de trabajo iniciará con la presentación de la demanda, de la cual conocerá la Junta Especial de la Federal de Conciliación

21 Ruiz Moreno, Ángel Guillermo, Nuevo derecho de la seguridad social, 14a. ed., México, Porrúa, 2011, p. 44.

22 Decreto por el que se reforman, adicionan y derogan diversas disposiciones de la Ley Federal del Trabajo, cit., p. 83.

23 Ibidem, pp. 83-86. 
Esta revista forma parte del acervo de la Biblioteca Jurídica Virtual del Instituto de Investigaciones Jurídicas de la UNAM

y Arbitraje del lugar en el que se encuentre la unidad de medicina familiar ${ }^{24}$ a la que estén adscritos los asegurados, cuando contenga — de acuerdo con el artículo 899-C de la reforma laboral— los requisitos siguientes:

- Nombre, domicilio, fecha de nacimiento del promovente y los documentos que acrediten su personalidad.

- La designación del perito médico o la solicitud por parte del asegurado para que le sea designado perito de acuerdo con el reformado artículo 824 de la Ley Federal del Trabajo (ante la falta de este requisito se le prevendrá al actor para que subsane la omisión en un término de tres días, apercibiéndolo de que en caso de no hacerlo se desechará de plano la demanda).

- La expresión de las pretensiones y de los hechos.

- El nombre y domicilio de las empresas en las que ha laborado el actor.

- Puestos y actividades desempeñados.

- Antigüedad.

- Número de seguridad social.

- Unidad de medicina familiar asignada.

- Los documentos expedidos por los patrones, el IMSS, el Instituto del Fondo Nacional de la Vivienda para los Trabajadores (Infonavit) y la administradora de fondos para el retiro (Afores) correspondiente o, en su caso, el acuse de recibo de la solicitud de los mismos, los cuales tienen la calidad de pruebas documentales que junto con las demás probanzas deben ofrecerse, pese a que en el artículo en cita se diferencian de manera innecesaria.

- Además, señalar las cotizaciones al régimen de seguridad social, adjuntar las copias de traslado y, en general, la información necesaria para la sustanciación del procedimiento. ${ }^{25}$

En torno al cúmulo de requisitos que deben cumplirse a fin de que la demanda sea admitida por la autoridad laboral, consideramos que al imponerse al asegurado la obligación de señalar el número de semanas cotizadas ante el régimen obligatorio del seguro social se contraviene lo dispuesto por el

24 Ibidem, p. 84.

25 Idem. 
Esta revista forma parte del acervo de la Biblioteca Jurídica Virtual del Instituto de Investigaciones Jurídicas de la UNAM

artículo 251 de la Ley del Seguro Social y el Reglamento de la Ley del Seguro Social en Materia de Afiliación, Clasificación de Empresas, Recaudación y Fiscalización, los cuales disponen que al IMSS le corresponde el registro e inscripción de los trabajadores para efectos del seguro social obligatorio, altas y bajas de éstos. De ahí que cuando existe controversia respecto del número de cotizaciones que servirán de base para el otorgamiento y cálculo de una pensión, su establecimiento y acreditamiento, tal y como lo dispone el artículo 899-D de la reforma realizada a la Ley Federal del Trabajo, ${ }^{26}$ debe recaer en el IMSS y no en el asegurado, para quien debería ser optativo el cumplimiento del requisito en análisis, al desconocer en la mayoría de los casos el tiempo que ha cotizado, además de carecer de los medios probatorios para computarlo y acreditarlo. ${ }^{27}$

Ante la admisión y radicación de la demanda, la JFCA señalará día y hora para la celebración de la audiencia de conciliación, demanda y excepciones, pruebas y resolución, en la cual primeramente la autoridad laboral, por conducto del funcionario conciliador o de su personal jurídico, intervendrá para la celebración de pláticas conciliatorias entre las partes y las exhortará para que procuren llegar a un acuerdo que dé por terminada la controversia planteada, pero en caso de no alcanzarse éste, cada una de las partes expondrá lo que juzgue conveniente, ofrecerá y desahogará las pruebas que hayan sido admitidas, teniendo un plazo de diez días hábiles, contados a partir de la celebración de la audiencia inicial, para que sus peritos acepten y protesten el cargo conferido y expresen en forma justificada los requerimientos necesarios para la emisión del dictamen pericial y la determinación del nexo causal tratándose de riesgos de trabajo, pues dentro de los treinta días siguientes a la celebración de la audiencia inicial, la JFCA señalará día y hora para la audiencia en la que, con citación de las partes, se recibirán los dictámenes periciales, ${ }^{28}$ los cuales deberán contener, de acuerdo con el artículo 899-E de la reforma laboral, los siguientes requisitos:

\footnotetext{
26 Idem.

27 El Instituto Mexicano del Seguro Social, en su calidad de órgano asegurador, cuenta con un registro manualizado, denominado Catálogo de Avisos Originales (CAO), y con un registro automatizado denominado Sistema Integral de Derechos y Obligaciones (SINDO), para determinar el número de semanas cotizadas por los asegurados.

28 Artículo 899-E del Decreto por el que se reforman, adicionan y derogan diversas disposiciones de la Ley Federal del Trabajo, cit., p. 85.
} 
Esta revista forma parte del acervo de la Biblioteca Jurídica Virtual del Instituto de Investigaciones Jurídicas de la UNAM

- Los datos de la identificación y de la acreditación de la profesión de médico de cada uno de los peritos.

- Los datos de identificación del actor, precisando el documento con el que se comprobó su identidad.

- El diagnóstico sobre los padecimientos reclamados.

- Tratándose de calificación y valuación de riesgos de trabajo, los razonamientos para determinar la relación de causa-efecto entre la actividad específica desarrollada por el trabajador y el estado de incapacidad cuya calificación o porcentaje de valuación se determine.

- Los medios de convicción en los cuales se basan las conclusiones del peritaje, incluyendo la referencia a los estudios médicos a los que se hubiera sometido el trabajador. ${ }^{29}$

Es importante no perder de vista que uno de los motivos de la reforma a la ley laboral fue combatir el anacronismo de las disposiciones procesales. ${ }^{30} \mathrm{Sin}$ embargo, estimamos que tal calificativo bien puede darse al hecho de constreñir al perito a acreditar estar autorizado ante la ley para ejercer la profesión de médico cirujano y partero cada vez que comparezca a rendir un dictamen médico cuando, por disposición del artículo 899-F de la referida reforma, se obliga a que todo perito médico que intervenga en los conflictos vinculados con la calificación y valuación de riesgos de trabajo y enfermedades generales necesariamente debe estar inscrito en el registro de la JFCA, ${ }^{31}$ lo cual sólo es procedente cuando entre otros requisitos acredite estar legalmente autorizado y capacitado para ejercer la profesión de médico.

En tales circunstancias, si se establece como una de las obligaciones de la JFCA el crear un registro de peritos médicos en materia de medicina del trabajo, en el cual sólo quedarán inscritos aquellos que acrediten estar legalmente autorizados y capacitados para ejercer la profesión de médico en un periodo de seis meses, contados a partir del establecimiento del registro correspondiente, y que ante el vencimiento de este plazo, la autoridad laboral

\footnotetext{
29 Idem.

30 30Secretaría del Trabajo y Previsión Social, Iniciativa de Decreto que reforma, adiciona y deroga diversas disposiciones de la Ley Federal del Trabajo, México, STPS, 2012, disponible en: bttp:/ / wmw. stps.gob.mx/bp/micrositios/reforma_laboral/ref_lab.htm [consultada el 11 de octubre de 2012].

31 Decreto por el que se reforman, adicionan y derogan diversas disposiciones de la Ley Federal del Trabajo, cit., p. 86.
} 
Esta revista forma parte del acervo de la Biblioteca Jurídica Virtual del Instituto de Investigaciones Jurídicas de la UNAM

no recibirá los dictámenes que emitan los peritos que carezcan de registro, resulta absurdo que en cada audiencia de desahogo de la prueba pericial, el perito tenga que acreditar estar autorizado para ejercer la profesión de médico, cuando se supone que al momento de registrarse la Junta certificó tal situación, pues de lo contrario el registro no hubiera procedido. ${ }^{32}$

Consideramos que al momento de rendir el dictamen médico, la autoridad laboral debería ir a la base de datos correspondiente y certificar si el galeno cuenta con registro o no, teniendo en el primero de los casos la obligación de recibir y agregar a los autos el correspondiente dictamen médico, sin necesidad de acreditación de la profesión de médico, ya que tal exigencia fue colmada al momento en que la JFCA autorizó su registro. En caso de que éste no se hubiera registrado o no hubiera procedido el registro, de acuerdo con el artículo décimo tercero transitorio de la reforma realizada a la Ley Federal del Trabajo, no se recibirán los dictámenes médicos emitidos por los peritos que carezcan de registro, ${ }^{33}$ aun cuando el profesionista acredite, en la audiencia de desahogo de la pericial, estar legalmente autorizado para ejercer la profesión sobre la que versará la prueba en cuestión. Esta última disposición contraviene el artículo 5o. constitucional, al impedírsele al perito el libre ejercicio de su profesión, como médico, para la cual está legalmente autorizado, tal y como lo acredita con la exhibición de su título y cédula profesional expedidos por la autoridad competente, además de no encontrarse dentro de las limitaciones impuestas a la libertad de trabajo en el texto constitucional y en el artículo $4 \mathrm{o}$. de la ley laboral.

32 En torno a este tema existe jurisprudencia bajo el rubro: "PERITO MÉDICO TERCERO EN DISCORDIA. Si ES OFICIAL, NO ESTÁ OBLIGADO EN TÉRMINOS DEL ARTÍCULO 822 DE LA Ley Federal del Trabajo a exhibir el título y CÉdula Profesional”, tesis I.3o.T. J/17, Semanario Judicial de la Federación y su Gaceta, Novena Época, mayo de 2005, t. XXI, la cual dispone que el artículo 822 de la Ley Federal del Trabajo prescribe que cuando se trate de ciencia o materia reglamentada, los peritos que intervengan en el juicio deben acreditar que tienen conocimientos sobre aquéllas y autorización para ejercer la profesión de que se trata. Sin embargo, aun cuando la medicina es una ciencia reglamentada, tratándose del perito tercero en discordia es innecesario que acredite en la audiencia respectiva esa formalidad, pues es evidente que dicho galeno, al ser nombrado por la Junta responsable, cuenta con la presunción legal de que previamente acreditó con título y cédula profesional que tiene conocimientos sobre la ciencia médica respecto de la que ha de dictaminar.

33 Decreto por el que se reforman, adicionan y derogan diversas disposiciones de la Ley Federal del Trabajo, cit., p. 91. 
Esta revista forma parte del acervo de la Biblioteca Jurídica Virtual del Instituto de Investigaciones Jurídicas de la UNAM

Ahora bien, por lo que hace al tercer requisito, reduce la emisión del dictamen médico al diagnóstico de los padecimientos reclamados por la parte actora, cuando consideramos que al contenerse en el dictamen médico la opinión de un especialista en el ramo médico en relación con las patologías del asegurado que solicita una pensión, debería determinarse la profesionalidad de todos y cada uno de los padecimientos que presente el asegurado y no avocarse únicamente a los demandados, ya que ante la presencia de un riesgo de trabajo es necesario que se "compense" el daño sufrido, evitándose, a su vez, que en un futuro el asegurado tenga que reclamar nuevamente los padecimientos que ya presentaba pero que no fueron calificados y valuados al no haberse señalado en una anterior demanda. Ante tal situación, el asegurado y el IMSS no verían mermado su patrimonio al tener que sufragar los gastos que conlleva un juicio laboral, reduciéndose, por su parte, para la autoridad laboral el número de asuntos a resolver.

A mayor abundamiento de lo expuesto, es de señalarse que el motivo por el cual la Junta solicita la rendición de dictámenes médicos es para que de una manera integral y objetiva la ilustren sobre el estado físico y psíquico del afectado, y en caso de determinarle algún tipo padecimiento establecer la naturaleza y la valuación de la lesión o el estado patológico que presenta, a fin de que la autoridad laboral cuente con elementos suficientes para resolver la controversia planteada.

El cuarto requisito que deben contener todos los dictámenes médicos que se rindan ante la JFCA consiste en la determinación por parte del perito médico de la relación de causa-efecto entre la actividad específica desarrollada por el trabajador y el estado de incapacidad, cuya calificación o valuación se determine; ${ }^{34}$ no corresponde con los tipos de riesgos de trabajo que contempla la Ley del Seguro Social.

Es claro que al exigirse en los riesgos de trabajo únicamente la acreditación de la relación de causa-efecto-daño entre las actividades desempeñadas por el asegurado y el padecimiento calificado como profesional, la reforma de la ley laboral olvida que éstos también pueden ser producto del medio ambiente en que el operario prestó sus servicios, motivo por el cual, en los dictámenes médicos, será necesario establecer el nexo causal entre el padecimiento y el medio ambiente. En el caso de que los padecimientos sean derivados de un

34 Ibidem, p. 85. 
Esta revista forma parte del acervo de la Biblioteca Jurídica Virtual del Instituto de Investigaciones Jurídicas de la UNAM

accidente de trabajo, el nexo causal debe establecerse entre la lesión y el accidente, así como precisarse el mecanismo y condiciones en que éste se realizó, aspectos todos ellos que la reforma dejó de considerar.

En cuanto al desahogo de la pericial médica, una vez que el perito emitió su dictamen, dispone el artículo 899-E de la reforma a la ley laboral que las partes y los miembros de la JFCA podrán hacer todo tipo de observaciones y formular preguntas al perito o a los peritos que comparezcan a la audiencia, en relación con las consideraciones y conclusiones establecidas en los correspondientes dictámenes, ${ }^{35}$ disposición que consideramos innecesaria ante la existencia de la fracción IV del reformado artículo 825 del código obrero, que prevé tal situación al estipular las reglas generales aplicables para el desahogo de la prueba pericial.

Finalmente, y de acuerdo con la fracción IV del artículo 895 de la ley laboral, se dispone que concluida la recepción de pruebas, la JFCA escuchará los alegatos y dictará la resolución. A este respecto consideramos que el artículo en comento debió reformarse para sustituir el término "recepción" por el de "desahogo", pues toda prueba —al ofrecerse conforme a derecho, tener relación con la litis planteada, no ser contraria a la moral y referirse a los hechos controvertidos $-{ }^{36}$ debe ser admitida por la autoridad laboral, quien deberá señalar día y hora para que tenga lugar su desahogo, en el caso que lo amerite, tal y como acontece en el caso de la pericial médica, siendo primeramente el "ofrecimiento", luego la "recepción" y finalmente el "desahogo" de las pruebas, tres aspectos totalmente diferentes que llevan una secuencia lógica dentro de todo procedimiento.

Una vez que el secretario de acuerdos de la JFCA certifique que no quedan pruebas pendientes por desahogar, procederá a escuchar los alegatos y acto seguido, al cierre de la instrucción, dictará resolución. Pese a nuestro escepticismo, en lo concerniente a este punto esperamos que se cumpla, por el bien de todos, terminándose con las largas esperas, de por lo menos ocho años, para que se dilucide una controversia en materia de riesgos de trabajo.

La tarea no va a resultar sencilla si tomamos en consideración que actualmente las juntas especiales 8, 8 Bis, 9 y 9 Bis de la JFCA que conocen de las

35 Idem.

36 Artículos 777, 779 y 780 de la Ley Federal del Trabajo, disponible en: http:/ / wmw.diputa dos.gob.mx/LeyesBiblio/index.htm [consultada el 18 de febrero de 2013]. 
Esta revista forma parte del acervo de la Biblioteca Jurídica Virtual del Instituto de Investigaciones Jurídicas de la UNAM

demandas interpuestas contra el IMSS tienen un rezago impresionante en cuanto al número de casos que cierran instrucción y se encuentran en el archivo esperando turno para ser resueltos por tan solo cuatro dictaminadores en las juntas especiales 9, 9 Bis y 8 Bis, y cinco dictaminadores en la Junta Especial 8, todas de la Federal de Conciliación y Arbitraje, quienes se encuentran resolviendo los asuntos correspondientes a los años 2006-2007, lo que se ha denominado "rezago histórico", estando además pendientes de resolver los años que corren de 2008 a la fecha, en que ingresan miles de demandas: tan sólo en 2011, de los 234,728 juicios individuales interpuestos, el 59\% de la carga de trabajo de la JFCA corresponde a los denominados conflictos de seguridad social en contra del IMSS, el Infonavit y las Afores. ${ }^{37}$

Si procedemos a distribuir el universo de asuntos en trámite, tenemos que el 31\% corresponde a asuntos del IMSS; 7\% a Pemex; 1\% a Ferrocarriles Nacionales de México en Liquidación e igual porcentaje a la extinta Luz y Fuerza del Centro (LyFC); 5\% a la Comisión Federal de Electricidad (CFE), y un estimado de $45 \%$ de juicios en contra de otros organismos públicos como el Infonavit, el Instituto de Seguridad y Servicios Sociales de los Trabajadores del Estado (ISSSTE), el Servicio Postal Mexicano (Sepomex) y las administradoras de fondos para el retiro (Afores), restando un 10\% en contra de empresas del sector privado. El 56\% del total de asuntos en trámite corresponde a conflictos de seguridad social. ${ }^{38}$ Aunado a ello, el gran cúmulo de trabajo de la JFCA, principalmente en la atención de asuntos relacionados con prestaciones de seguridad social, ha generado el incremento de amparos por omisión de cumplimiento de términos legales, por lo que para evitar incidentes de inejecución de sentencia de amparo deben resolverse en forma inmediata algunos expedientes más recientes, en detrimento de los del rezago histórico. ${ }^{39}$

Por su parte, de acuerdo con la base estadística que las 66 juntas especiales que componen la Federal de Conciliación y Arbitraje, se reportan al 31 de julio de 2013 los siguientes datos: ${ }^{40}$

37 Junta Federal de Conciliación y Arbitraje, Informe anual de labores 2012, México, JFCA, 2012, consultable en: http://wmw.stps.gob.mx/bp/secciones/junta_federal/secciones/documentos/IN FORME_ANUAL_DE_LABORES_2012.pdf [consultada el 11 de octubre de 2013].

38 Idem.

39 Idem.

40 Información proporcionada el 20 de agosto de 2013 por la Dirección de Evaluación, Coordinación General de Administración de la Junta Federal de Conciliación y Arbitraje. 
Esta revista forma parte del acervo de la Biblioteca Jurídica Virtual del Instituto de Investigaciones Jurídicas de la UNAM

NÚMERO TOTAL DE CONFLICTOS INDIVIDUALES DE SEGURIDAD SOCIAL INTERPUESTOS AL 31 DE JULIO DE 2013

Institución

IMSS

Afores

Infonavit

Otros

Carga de trabajo

(Instrucción + dictamen)

Fase de amparo

Fase de laudo y ejecución

Total

\begin{tabular}{|l|}
\multicolumn{1}{|l}{$206,018(53.1 \%)$} \\
\hline IMSS, Afores e Infonavit \\
- 48,615 Laudo \\
- 13,548 Ejecución \\
\hline
\end{tabular}

74,322 juicios se reclaman prestaciones relacionadas con la devolución del fondo de la subcuenta de vivienda 97, desglosados:

— 44,337 Instrucción y dictamen

— 29,985 Laudo y ejecución
Laboral

$13,402^{-1}(20.0 \%)$

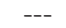

$836(11.0 \%)$

$97,211(100 \%)$

$111,449(50.9 \%)$

$40,988(81.4 \%)$

$53,581(45.1 \%)$

206,018 (53.1\%)
Seguridad social, Afores y fondo de vivienda

$53,603(80.0 \%)$

$47,319^{-2}(100 \%)$

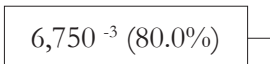

7,586

97,211

219,121

50,365

118,744

388,230

$182,212(46.9 \%)$

67,005

107,672 (49.1\%)

9,377 (18.6\%)

65,163 (54.9\%)

Total

67,005

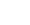


Esta revista forma parte del acervo de la Biblioteca Jurídica Virtual del Instituto de Investigaciones Jurídicas de la UNAM

Empero, si bien las reformas a la Ley Federal del Trabajo plantean un nuevo procedimiento para la pronta resolución de los juicios relacionados con las prestaciones derivadas de la Ley del Seguro Social — procedimiento acompañado de una serie de requisitos que debe cumplir el perito médico y los dictámenes que emitan, así como reglas referentes al desahogo de la pericial médica (las cuales, como analizamos, son incompletas, innecesarias y en algunos casos absurdas)—, adolecen de un procedimiento de calificación de los riesgos de trabajo, ya que consideramos que para que éste exista se debe regular la serie de actos involucrados en lograr dicho fin, además de brindarse la oportunidad para que el asegurado presente en un plazo determinado en los Servicios de Salud en el Trabajo del IMSS aquellas probanzas que acrediten los padecimientos profesionales que arguye tener, lo anterior en aras de lograr una mayor imparcialidad en el dictado de resoluciones administrativas, evitándose así las miles de demandas interpuestas.

Es ante la inexistencia en la Ley del Seguro Social y en la Ley Federal del Trabajo de un procedimiento para la calificación de un riesgo como profesional que se deja en manos de los facultativos de Salud en el Trabajo del IMSS o a los peritos médicos que comparecen ante las autoridades laborales, la ardua tarea de calificar y valorar los padecimientos, permitiéndoles interpretar a su manera los preceptos legales y los alcances del espíritu regulador de la norma, pese a que no cuentan con la preparación técnica ni académica para ello, dado que su especialidad es la ciencia médica y no la jurídica. La pregunta lógica es ¿por qué a más de medio siglo de operar el IMSS y ante la reforma laboral de 2012 no se ha reglamentado el procedimiento para la calificación de profesionalidad de un riesgo?

Consideramos que la respuesta obedece a que existen fuertes intereses creados sobre este particular, que han dejado en manos de los médicos el dictamen de profesionalidad de los riesgos ocurridos, cuya emisión se torna una tarea complicada ante la conjugación de los siguientes factores: las incompletas, desactualizadas e inadecuadas tablas de enfermedades de trabajo y de valuación de incapacidades permanentes previstas en los artículos 513 y 514 de la ley laboral, ${ }^{41}$ y la negligencia de las autoridades correspondientes para

41 El artículo cuarto transitorio de la Ley Federal del Trabajo establece que la STPS deberá expedir las tablas de enfermedades de trabajo y de valuación de incapacidades permanentes resultantes de los riesgos de trabajo, en un término de seis meses contados a partir de la entrada 
Esta revista forma parte del acervo de la Biblioteca Jurídica Virtual del Instituto de Investigaciones Jurídicas de la UNAM

actualizarlas periódicamente. En vista de los avances científicos, los cambios tecnológicos, los nuevos procedimientos de trabajo y la mundialización, resulta prioritario el reconocimiento de las nuevas enfermedades profesionales y de los criterios para identificarlas.

Asimismo, ante tan importante misión que se encomienda a los médicos en el dictamen de la profesionalidad de un accidente o enfermedad, se torna imprescindible que los facultativos posean los conocimientos indispensables y la acreditación de estar autorizados conforme a la ley para la práctica de la especialidad en medicina del trabajo con la que deberían contar y no sólo tener tres años de experiencia profesional vinculada con la medicina del trabajo tal y como lo dispone el artículo 899-F de la reforma laboral. ${ }^{42}$ Además, deberían conocer, dominar y aplicar las técnicas específicas de su trabajo; comprender la estructura y la dinámica socioeconómica-cultural de la sociedad a la que pertenecen, así como estar sujetos a una constante capacitación, pues su labor exige un amplio conocimiento de las causas que dan lugar a la toxicología y patología del trabajo, debiéndose tomar en cuenta diversos factores, tales como el grado de la contaminación del ambiente de trabajo, el tiempo de exposición, las medidas preventivas que fueron puestas en práctica, los casos análogos, los estudios clínicos y las visitas armadas.

En tales condiciones, y toda vez que la mayor parte de la reforma a la Ley Federal del Trabajo se refiere al procedimiento para interponer, tramitar y resolver los conflictos individuales de seguridad social, lamentablemente la problemática analizada con antelación sigue presente, en espera de que se reforme la Ley del Seguro Social o se apruebe una nueva que busque establecer en nuestro país la tan anhelada seguridad social.

\section{BIBLIOGRAFÍA}

BRICEÑo Ruiz, Alberto, Derecho de la seguridad social, México, Oxford University Press, 2011.

en vigor del referido decreto, motivo por el cual se seguirán aplicando las desactualizadas e incompletas tablas previstas en los artículos 513 y 514 de la anterior ley laboral.

42 Decreto por el que se reforman, adicionan y derogan diversas disposiciones de la Ley Federal del Trabajo, cit., p. 86. 
Esta revista forma parte del acervo de la Biblioteca Jurídica Virtual del Instituto de Investigaciones Jurídicas de la UNAM

Buen Lozano, Néstor de, Manual de derecho de la seguridad social, México, Porrúa, 2006.

García Maldonado, Octavio, Teoría y práctica de la seguridad social, México, Centro Universitario de Ciencias Sociales y Humanidades, 2003.

Instituto Mexicano de Contadores Públicos, Riesgos de trabajo. Obligaciones y derechos de los patrones. Estudio integral del seguro de riesgos de trabajo, México, Porrúa, 2007.

Instituto Mexicano del Seguro Social, "Informe al Ejecutivo Federal y al Congreso de la Unión sobre la situación financiera y los riesgos del Instituto Mexicano del Seguro Social 2012-2013”, México, IMSS, 2012-2013, disponible en: http://wnw.imss.gob.mx/estadisticas/Documentos/20122013/in formecompleto.pdf [consultada el 11 de octubre de 2013].

instituto Nacional de Estadística y Geografía, Censo de Población y Vivienda 2010, México, INEGI, disponible en: http://mmw.inegi.org.mx/ default.aspx? [consultada el 11 de octubre de 2013].

Kelsen, Hans, Teoría pura del derecho, 10a. ed., trad. de Roberto J. Vernengo, México, Porrúa, 1998.

MendizÁBAl Bermúdez, Gabriela, La seguridad social en México, México, Porrúa, 2007.

ORGANIZACIÓN INTERNACIONAL DEL TRABAJO, Lista de enfermedades profesionales (revisada en 2010). Identificación y reconocimiento de las enfermedades profesionales: criterios para incluir enfermedades en la lista de enfermedades profesionales de la OIT, Ginebra, Oficina Internacional del Trabajo, 2010, Serie Seguridad y Salud en el Trabajo, núm. 74.

Ramos Ruvalcaba, María Simona y Díaz Rivadeneyra, José Carlos, Nueva Ley del Seguro Social comentada. Orientación práctica, México, Porrúa, 2007.

Ruiz Moreno, Ángel Guillermo, Las afore: el sistema de ahorro y pensiones, 6 a. ed., México, Porrúa, 2009.

—, Nuevo derecho de la seguridad social, 14a. ed., México, Porrúa, 2011.

SENIOR F., Alberto, Un curso de filosofía del derecho, México, s.e., 1999. 